\title{
Sound Propagation in a Duct with Wall Corrugations Having Square-Wave Profiles
}

\author{
Muhammad A. Hawwa \\ Department of Mechanical Engineering, King Fahd University of Petroleum and Minerals, Dhahran 31261, Saudi Arabia \\ Correspondence should be addressed to Muhammad A. Hawwa; drmafh@kfupm.edu.sa
}

Received 14 April 2015; Revised 17 June 2015; Accepted 18 June 2015

Academic Editor: Hakim Naceur

Copyright (C) 2015 Muhammad A. Hawwa. This is an open access article distributed under the Creative Commons Attribution License, which permits unrestricted use, distribution, and reproduction in any medium, provided the original work is properly cited.

Acoustic wave propagation in ducts with rigid walls having square-wave wall corrugations is considered in the context of a perturbation formulation. Using the ratio of wall corrugation amplitude to the mean duct half width, a small parameter is defined and a two levels of approximations are obtained. The first-order solution produces an analytical description of the pressure field inside the duct. The second-order solution yields an analytical estimate of the phase speed of waves transmitting through the duct. The effect of wall corrugation density on acoustic impedance and wave speeds is highlighted. The analysis reveals that waves propagating in a duct with square-wave wall corrugation are slower than waves propagating in a duct with sinusoidal wave corrugation having the same corrugation wavelength.

\section{Introduction}

Acoustic waveguides with walls having weak periodic undulations have been modules of interest over the past decades. Although wall undulations constitute small features at the boundaries of the wave propagation domain, they can significantly transform acoustic wave propagation characteristics. Wall periodicities are commonly added patterned (periodic) corrugations that increase structural strength while keeping duct flexibility. In another scenario situation, geometric roughness on a duct wall can be looked at as irregular corrugations. Regardless, whether wall corrugations are deterministically periodic or statistically having a mean periodic profile, the dispersion spectrum of periodic acoustic waveguide is divided into passbands and stopbands.

As first steps towards dealing with the case of a waveguide with statistically rough boundaries, Samuels [1] and then Salant [2] employed a straightforward perturbation expansion for case of sound propagation in a two-dimensional (parallel plate) waveguide with small sinusoidal perturbations in the boundaries to study forward- and backwardpropagating mode coupling. Nayfeh [3] employed a multiple scales perturbation technique to show that the perturbation expansion employed by Samuels [1] and Salant [2] was not uniform under resonance conditions and provided a uniformly valid perturbation expansion. Nusayr [4] considered the mode coupling case in a rectangular waveguide with sinusoidal boundaries, employing a multiple scales perturbation technique in the analysis. Boström [5] utilized a null field approach to study sound propagation in a cylindrical duct whose radius varied sinusoidally along its axial dimension and proved the existence of small passbands for the case of large-amplitude undulations. Asfar and Nayfeh [6] provided a review on using the perturbation method of multiple scales for wave propagation in periodic structures, including closedand open-acoustic waveguides. Lundqvist and Boström [7] calculated transmission and reflection coefficients at the junction between a straight and a sinusoidally corrugated cylindrical duct using mode-matching under the context of a null field approach.

After 1990, Bradley [8] found Bloch wave functions to describe waves in a rectangular duct having rectangular side branches placed periodically and presented strong dispersion and sharply banded attenuation that could be used in a band reject travelling wave filter. Hawwa [9] presented a multiple scales perturbation approach for analyzing waveguides with surfaces having two periodicities and detailed scenarios of various incidents and reflected mode coupling in these 
waveguides. Hawwa [10] examined the influence of weighted tapered periodic undulations on acoustic wave reflection spectra in waveguides using perturbation analysis. Hawwa [11] investigated the effect of chirped periodic surface undulations on widening stopbands of acoustic wave reflection spectra in ducts by solving coupled mode equations describing modal interactions. Tao et al. [12] made a predictive study of non-Bragg resonance, caused by the interaction between the transverse standing acoustic waves occurring in a cylindrical waveguide with a sinusoidally perturbed wall. Potel and Bruneau [13] used standard integral Green's formulation to describe the nonlocal mode coupling in an inviscid fluid-filled waveguide enclosed between two planes having two-dimensional irregular corrugations. Valier-Brasier et al. [14] considered acoustic eigenmode coupling in a fluidfilled waveguide enclosed between two planes having onedimensional irregular corrugations having the shape of tiny parallel ridges. Tao et al. [15] showed the possibility of acoustic energy transmission within a Bragg stopband by means of high-order transverse modes interactions in an acoustic duct with periodically corrugated walls. Del Giudice and Bernasconi [16] utilized an acoustic horn equation to get reflection and transmission spectra in a waveguide with small sinusoidal cross section variation. Tao and Fan [17] used a perturbation method and a finite element formulation to consider transmission under non-Bragg resonances of three transverse modes in a waveguide with sinusoidally perturbed walls.

Almost all of the works cited above are concerned with sound propagating in waveguides with periodic wall corrugations and concentrate on wave modal interactions under Bragg and non-Bragg conditions. In this paper, focus is placed on acoustic wave transmission through a two-dimensional waveguide with wall corrugations having the geometry of square waves. The work intends to describe the acoustic field in terms of pressure and impedance along this type of periodic ducts, estimate the phase speed of transmitted wave at frequencies which lies within passbands, and compare the speed of sound in this type of waveguides to that in a duct with sinusoidal corrugations. Considering small corrugation amplitude, a small parameter is defined from the ratio of the corrugation amplitude and the waveguide average thickness and the perturbation method of strained parameters is used up to the second order. The influence of wall corrugation density is considered as a differentiating factor for graphical presentation of obtained analytical results.

\section{Mathematical Model and Solution}

A two-dimensional acoustic waveguide (duct) is confined between two rigid walls having square-wave geometries, as shown in Figure 1. The mass velocity of air contained in the waveguide is much smaller than the speed of sound in this inviscid medium. Acoustic waves are considered in the form of perturbations of the medium pressure $\widetilde{p}(\vec{x}, t)$, density $\widetilde{\rho}(\vec{x}, t)$, and mass velocity $\widetilde{\vec{v}}(\vec{x}, t)$, where $t$ is the time coordinate. The linearized continuity and momentum conservation equations are supplemented with a barotropic fluid equation of state to yield the following wave equations:

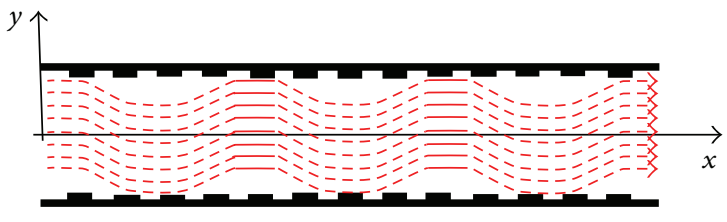

FIGURE 1: The two-dimensional periodic acoustic duct.

$$
\begin{aligned}
& \nabla^{2} \widetilde{p}=\frac{1}{c_{0}^{2}} \frac{\partial^{2} \tilde{p}}{\partial t^{2}}, \\
& \nabla^{2} \widetilde{\rho}=\frac{1}{c_{0}^{2}} \frac{\partial^{2} \widetilde{\rho}}{\partial t^{2}}, \\
& \nabla^{2} \widetilde{\vec{v}}=\frac{1}{c_{0}^{2}} \frac{\partial^{2} \widetilde{\vec{v}}}{\partial t^{2}},
\end{aligned}
$$

where $c_{0}^{2}\left(=\gamma p_{0} / \rho_{0}\right)$ is the speed of sound, $\left(p_{0}, \rho_{0}\right)$ represent the spatially uniform state, and $\gamma$ is the specific heat ratio of the isentropic gas.

Based on momentum conservation, a velocity potential $\Phi$ can be defined such that $\widetilde{\vec{v}}=\nabla \widetilde{\Phi}$, while the acoustic pressure can be calculated from $\widetilde{p}=-\rho_{0}(\partial \widetilde{\Phi} / \partial \widetilde{t})$; then the wave equation can be written as

$$
\nabla^{2} \widetilde{\Phi}=\frac{\partial^{2} \widetilde{\Phi}}{\partial \widetilde{x}^{2}}+\frac{\partial^{2} \widetilde{\Phi}}{\partial \widetilde{y}^{2}}=\frac{1}{c_{0}^{2}} \frac{\partial^{2} \widetilde{\Phi}}{\partial \widetilde{t}^{2}} .
$$

This equation is complemented by the boundary condition of the vanishing of the velocity component normal to the duct walls; hence

$$
\begin{aligned}
\frac{\partial \widetilde{\Phi}}{\partial \widetilde{x}} v_{x}+\frac{\partial \widetilde{\Phi}}{\partial \widetilde{y}} v_{y} & =0 \quad \text { at lower wall, } \\
\widetilde{y}(\widetilde{x}) & =-h-a \sum_{j=1,3,5, \ldots} \frac{1}{j} \sin \left(\widetilde{k}_{w} \widetilde{x}\right), \\
\frac{\partial \widetilde{\Phi}}{\partial x} v_{x}+\frac{\partial \widetilde{\Phi}}{\partial y} v_{y} & =0 \quad \text { at upper wall, } \\
\tilde{y}(\tilde{x}) & =h+\delta a \sum_{j=1,3,5, \ldots} \frac{1}{j} \sin \left(\widetilde{k}_{w} \tilde{x}+\theta\right),
\end{aligned}
$$

where $\left\langle v_{x}, v_{y}\right\rangle$ are the components of unit outward-pointing vector normal to the wall. Note that the square-wave geometries are approximated using Fourier series expansions. Also, note that $2 h$ is the average width of the duct, $\widetilde{k}_{w}$ is the wavenumber of the duct wall corrugation, $a$ is the amplitude of corrugation, $\theta$ is a phase angle between the two wall corrugations, and $\alpha$ is a coefficient allowing a different corrugation amplitude. This way of describing the duct wall has the advantage of getting a solution of the case of duct with sinusoidally corrugated walls as a special case; it also gives a chance for describing imperfect or nearly square wave by limiting the number of terms under the summation. 
Assume that the acoustic waves along the duct propagate as time-harmonic signals such that $\widetilde{\Phi}=\widetilde{\phi}(\widetilde{x}, \tilde{y}) e^{-i \omega \widetilde{t}}$, where $\widetilde{\phi}$ is the velocity potential amplitude and $\omega$ is the angular frequency. Then, let half of the average width of the 2dimensional duct $h$ be a spatial reference quantity and let $\omega^{-1}$ be a temporal reference quantity. The governing equation of acoustic waves in the duct and the boundary conditions can then be written in the following dimensionless form:

$$
\begin{aligned}
& \frac{\partial^{2} \phi}{\partial x^{2}}+\frac{\partial^{2} \phi}{\partial y^{2}}+\left(\frac{\omega h}{c_{0}}\right)^{2} \phi=0, \\
& \frac{\partial \phi}{\partial y}=-\varepsilon k_{w} \sum_{j=1,3,5, \ldots} \frac{1}{j} \cos \left(k_{w} x\right) \frac{\partial \phi}{\partial x} \\
& \text { at } y=-1-\varepsilon \sum_{j=1,3,5, \ldots} \frac{1}{j} \sin \left(k_{w} x\right), \\
& \frac{\partial \phi}{\partial y}=\varepsilon \delta k_{w} \sum_{j=1,3,5, \ldots} \frac{1}{j} \cos \left(k_{w} x+\theta\right) \frac{\partial \phi}{\partial x} \\
& \text { at } y=1+\delta \varepsilon \sum_{j=1,3,5, \ldots} \frac{1}{j} \sin \left(k_{w} x+\theta\right),
\end{aligned}
$$

where $\varepsilon(=a / h)$ is a small dimensionless parameter that is equal to the ratio of the amplitude of the wall corrugation to the average half width of the duct.

The inhomogeneous boundary conditions (3b) and (3c) can be expanded using Taylor series around $y=-1$ and $y=+1$, respectively. This helps in transferring the boundary conditions to the average line of the square-wave geometries. Then, one has to deal with the following system of equations:

$$
\begin{aligned}
& \frac{\partial^{2} \phi}{\partial x^{2}}+\frac{\partial^{2} \phi}{\partial y^{2}}+k^{2} \phi=0, \\
& \frac{\partial \phi}{\partial y}(x,-1)-\varepsilon\left[\sum_{j=1,3,5, \ldots} \frac{1}{j} \sin \left(k_{w} x\right)\right] \frac{\partial^{2} \phi}{\partial y^{2}}(x,-1) \\
& +\frac{1}{2} \varepsilon^{2}\left[\sum_{j=1,3,5, \ldots} \frac{1}{j} \sin \left(k_{w} x\right)\right]^{2} \frac{\partial^{3} \phi}{\partial y^{3}}(x,-1)+\cdots \\
& =-\varepsilon\left[k_{w} \sum_{j=1,3,5, \ldots} \frac{1}{j} \cos \left(k_{w} x\right)\right]\left\{\frac{\partial \phi}{\partial x}(x,-1)\right. \\
& \quad-\varepsilon\left[\sum_{j=1,3,5, \ldots} \frac{1}{j} \sin \left(k_{w} x\right)\right] \frac{\partial^{2} \phi}{\partial x \partial y}(x,-1) \\
& +\frac{1}{2} \varepsilon^{2}\left[\sum_{j=1,3,5, \ldots} \frac{1}{j} \sin \left(k_{w} x\right)\right]^{2} \frac{\partial^{3} \phi}{\partial x \partial y^{2}}(x,-1) \\
& +\cdots\},
\end{aligned}
$$

$$
\begin{aligned}
& \frac{\partial \phi}{\partial y}(x, 1)+\varepsilon\left[\delta \sum_{j=1,3,5, \ldots} \frac{1}{j} \sin \left(k_{w} x+\theta\right)\right] \frac{\partial^{2} \phi}{\partial y^{2}}(x, 1) \\
& +\frac{1}{2} \varepsilon^{2}\left[\delta \sum_{j=1,3,5, \ldots} \frac{1}{j} \sin \left(k_{w} x+\theta\right)\right]^{2} \frac{\partial^{3} \phi}{\partial y^{3}}(x, 1) \\
& +\cdots=\varepsilon\left[\delta k_{w} \sum_{j=1,3,5, \ldots} \frac{1}{j} \cos \left(k_{w} x+\theta\right)\right] \\
& .\left\{\frac{\partial \phi}{\partial x}(x, 1)\right. \\
& +\varepsilon\left[\delta \sum_{j=1,3,5, \ldots} \frac{1}{j} \sin \left(k_{w} x+\theta\right)\right] \frac{\partial^{2} \phi}{\partial x \partial y}(x, 1) \\
& +\frac{1}{2} \varepsilon^{2}\left[\delta \sum_{j=1,3,5, \ldots} \frac{1}{j} \sin \left(k_{w} x+\theta\right)\right]^{2} \frac{\partial^{3} \phi}{\partial x \partial y^{2}}(x, 1) \\
& +\cdots\} .
\end{aligned}
$$

Note that (4a) is a standard Helmholtz wave equation and $k\left(=\omega h / c_{0}\right)$ is the nondimensional wavenumber, while the boundary conditions (4b) and (4c) are given two levels of approximation.

2.1. Asymptotic Formulation. In order to solve the system of (4a)-(4c), the perturbation method of strained parameters [18] is used for determining more accurate approximations in a successive fashion. The reason for selecting this perturbation method is to account for the fact that when an acoustic signal propagates in the geometrically inhomogeneous duct its wavenumber becomes a function in the parameter measuring the inhomogeneity. Thus, $\phi$ and $k$ are expanded in powers of $\varepsilon$ :

$$
\begin{aligned}
& \phi=\varepsilon^{0} \phi_{0}+\varepsilon^{1} \phi_{1}+\varepsilon^{2} \phi_{2}+\cdots, \\
& k=\varepsilon^{0} k_{0}+\varepsilon^{1} \varepsilon k_{1}+\varepsilon^{2} k_{2}+\cdots .
\end{aligned}
$$

Substituting (5) into (4a), (4b), and (4c) and equating coefficients of equal powers on both sides of the equations, one obtains a zeroth-order, a first-order, and a second-order problem as follows:

$$
\begin{aligned}
& \text { O(1) Problem } \\
& \begin{aligned}
\frac{\partial^{2} \phi_{0}}{\partial x^{2}}+\frac{\partial^{2} \phi_{0}}{\partial y^{2}}+k_{0}^{2} \phi_{0} & =0, \\
\frac{\partial \phi_{0}}{\partial y} & =0, \quad \text { At } y=0, \\
\frac{\partial \phi_{0}}{\partial y} & =0, \quad \text { At } y=1 .
\end{aligned}
\end{aligned}
$$




\section{$O(\varepsilon)$ Problem}

$$
\begin{aligned}
& \frac{\partial^{2} \phi_{1}}{\partial x^{2}}+\frac{\partial^{2} \phi_{1}}{\partial y^{2}}+k_{0}^{2} \phi_{1}=-k_{1}^{2} \phi_{0}-2 k_{0} k_{1} \phi_{0}, \\
& \frac{\partial \phi_{1}}{\partial y} \\
& =\left[\sum_{j=1,3,5, \ldots} \frac{1}{j} \sin \left(k_{w} x\right)\right] \frac{\partial^{2} \phi_{0}}{\partial y^{2}} \\
& \quad-\left[k_{w} \sum_{j=1,3,5, \ldots} \frac{1}{j} \cos \left(k_{w} x\right)\right] \frac{\partial \phi_{0}}{\partial x}, \quad \text { at } y=-1,
\end{aligned}
$$

$\frac{\partial \phi_{1}}{\partial y}$

$$
\begin{aligned}
= & -\left[\delta \sum_{j=1,3,5, \ldots} \frac{1}{j} \sin \left(k_{w} x+\theta\right)\right] \frac{\partial^{2} \phi_{0}}{\partial y^{2}} \\
& +\left[\delta k_{w} \sum_{j=1,3,5, \ldots} \frac{1}{j} \cos \left(k_{w} x+\theta\right)\right] \frac{\partial \phi_{0}}{\partial x},
\end{aligned}
$$

at $y=1$.

$$
O\left(\varepsilon^{2}\right) \text { Problem }
$$

$$
\begin{aligned}
& \frac{\partial^{2} \phi_{2}}{\partial x^{2}}+\frac{\partial^{2} \phi_{2}}{\partial y^{2}}+k_{0}^{2} \phi_{2}=-\left(k_{1}^{2}+2 k_{0} k_{2}\right) \phi_{0}-\left(2 k_{0} k_{1}\right) \\
& \cdot \phi_{1}, \\
& \frac{\partial \phi_{2}}{\partial y}=\left[\sum_{j=1,3,5, \ldots} \frac{1}{j} \sin \left(k_{w} x\right)\right] \\
& \cdot\left\{\frac{\partial^{2} \phi_{1}}{\partial y^{2}}-\frac{1}{2}\left[\sum_{j=1,3,5, \ldots} \frac{1}{j} \sin \left(k_{w} x\right)\right] \frac{\partial^{3} \phi_{0}}{\partial y^{3}}\right\} \\
& -\left[k_{w} \sum_{j=1,3,5, \ldots} \frac{1}{j} \cos \left(k_{w} x\right)\right] \\
& \cdot\left\{\frac{\partial \phi_{1}}{\partial x}-\left[\sum_{j=1,3,5, \ldots} \frac{1}{j} \sin \left(k_{w} x\right)\right] \frac{\partial^{2} \phi_{0}}{\partial x \partial y}\right\}
\end{aligned}
$$$$
\text { at } y=-1 \text {, }
$$

$$
\begin{aligned}
& \frac{\partial \phi_{2}}{\partial y}=-\left[\delta \sum_{j=1,3,5, \ldots} \frac{1}{j} \sin \left(k_{w} x+\theta\right)\right] \\
& \cdot\left\{\frac{\partial^{2} \phi_{1}}{\partial y^{2}}-\frac{1}{2}\left[\delta \sum_{j=1,3,5, \ldots} \frac{1}{j} \sin \left(k_{w} x+\theta\right)\right] \frac{\partial^{3} \phi_{0}}{\partial y^{3}}\right\}
\end{aligned}
$$

$$
\begin{aligned}
& +\left[\delta k_{w} \sum_{j=1,3,5, \ldots} \frac{1}{j} \cos \left(k_{w} x+\theta\right)\right] \\
& \cdot\left\{\frac{\partial \phi_{1}}{\partial x}+\left[\delta \sum_{j=1,3,5, \ldots} \frac{1}{j} \sin \left(k_{w} x+\theta\right)\right] \frac{\partial^{2} \phi_{0}}{\partial x \partial y}\right\}
\end{aligned}
$$$$
\text { at } y=1 \text {. }
$$

2.2. Zeroth-Order Solution. As with any perturbation technique, a systematic procedure for determining successively more accurate approximations is followed. Starting with the zeroth-order problem, let us select a mode with a frequency that lies in the passband part of the banded spectrum. Then, a solution of (6a)-(6c) is sought in terms of a forward-traveling acoustic mode, $n$, as

$$
\phi_{0}(x, y)=A_{n} \cos (n \pi y) e^{i k_{n} x},
$$

where $k_{n}$ is the wavenumber of the propagation acoustic mode. The substitution of the assumed solution (9) into (4b) and $(4 \mathrm{c})$ leads to the following dispersion relation:

$$
k_{n}=\sqrt{k_{0}^{2}-(n \pi)^{2}} .
$$

2.3. First-Order Solution. Next, let us solve the first-order problem. A particular solution for $\phi_{1}$ can be postulated in the following form:

$$
\begin{aligned}
\phi_{1}(x, y)= & \varphi_{1}(y) e^{i k_{n} x}+\varphi_{2}(y) e^{i\left(k_{n}+k_{w}\right) x} \\
& +\varphi_{3}(y) e^{i\left(k_{n}-k_{w}\right) x} .
\end{aligned}
$$

Substituting (9) and (11) into the governing equations (7a), (7b), and (7c) and equating the coefficients of $e^{i k_{n} x}, e^{i\left(k_{n}+k_{w}\right) x}$, and $e^{i\left(k_{n}-k_{w}\right) x}$ yield three problems in $\varphi_{1}, \varphi_{2}$, and $\varphi_{3}$, as follows:

$$
\begin{aligned}
& \varphi_{1}^{\prime \prime}(y)+(n \pi)^{2} \varphi_{1}(y) \\
& =-\left[k_{1}^{2}+2 k_{0} k_{1}\right] A_{n} \cos (n \pi y), \\
& \frac{\partial \varphi_{1}}{\partial y}(-1)=0, \\
& \frac{\partial \varphi_{1}}{\partial y}(1)=0,
\end{aligned}
$$

$$
\varphi_{2}^{\prime \prime}(y)+\left[k_{0}^{2}-\left(k_{n}+k_{w}\right)^{2}\right] \varphi_{2}(y)=0,
$$

$$
\frac{\partial \varphi_{2}}{\partial y}(-1)
$$

$$
=-\left[\sum_{j=1,3,5, \ldots} \frac{i}{2 j}\right]\left[(n \pi)^{2}+k_{n} k_{w}\right] A_{n} \cos (-n \pi),
$$




$$
\begin{aligned}
& \frac{\partial \varphi_{2}}{\partial y}(1) \\
& =\left[\sum_{j=1,3,5, \ldots} \frac{i}{2 j}\right]\left[(n \pi)^{2}+k_{w} k_{n}\right] A_{n} \cos (n \pi) \delta e^{i \theta}, \\
& \varphi_{3}^{\prime \prime}(y)+\left[k_{0}^{2}-\left(k_{n}-k_{w}\right)^{2}\right] \varphi_{3}(y)=0, \\
& \frac{\partial \varphi_{3}}{\partial y}(-1) \\
& =\left[\sum_{j=1,3,5, \ldots} \frac{i}{2 j}\right]\left[(n \pi)^{2}-k_{n} k_{w}\right] A_{n} \cos (-n \pi), \\
& \frac{\partial \varphi_{3}}{\partial y}(1) \\
& =-\left[\sum_{j=1,3,5, \ldots} \frac{i}{2 j}\right]\left[(n \pi)^{2}-k_{n} k_{w}\right] A_{n} \cos (n \pi) \delta e^{-i \theta} .
\end{aligned}
$$

In order to solve (12a)-(12c), we multiply the governing equation (12a) by $\cos (j \pi y)$ and integrate by parts from $y=-1$ to $y=+1$; then we invoke the boundary conditions (12b) and (12c), which lead to $k_{1}=0$. Hence, there is no first-order correction for the wavenumber and a higher-order correction is needed to describe the effect of surface undulations on the propagation characteristics.

The governing equation (13a) and (14a), respectively, admit solutions of the form

$$
\begin{aligned}
\varphi_{2}(y) & =b_{21} e^{i p_{2} y}+b_{22} e^{-i p_{2} y} \quad \text { where } \\
p_{2} & =\sqrt{k_{0}^{2}-\left(k_{n}+k_{w}\right)^{2}} \\
\varphi_{3}(y) & =b_{31} e^{i p_{3} y}+b_{32} e^{-i p_{3} y} \quad \text { where } \\
p_{3} & =\sqrt{k_{0}^{2}-\left(k_{n}-k_{w}\right)^{2}} .
\end{aligned}
$$

Substituting solution (15) into the boundary conditions (13b) and (13c) and solution (16) into the boundary conditions (14b) and (14c) leads to determining the unknown coefficients as

$$
\begin{aligned}
& \left\{\begin{array}{l}
b_{21} \\
b_{22}
\end{array}\right\}=\frac{\cos (n \pi)}{4 p_{2} \sin \left(2 p_{2}\right)}\left[\sum_{j=1,3,5, \ldots} \frac{1}{2 j}\right]\left[(n \pi)^{2}+k_{n} k_{w}\right] \\
& .\left\{\begin{array}{l}
e^{-i p_{2}}+e^{i p_{2}} \delta e^{i \theta} \\
e^{i p_{2}}+e^{-i p_{2}} \delta e^{i \theta}
\end{array}\right\} A_{n}, \\
& \left\{\begin{array}{l}
b_{31} \\
b_{32}
\end{array}\right\}=-\frac{\cos (n \pi)}{4 p_{3} \sin \left(2 p_{3}\right)}\left[\sum_{j=1,3,5, \ldots} \frac{1}{2 j}\right]\left[(n \pi)^{2}-k_{n} k_{w}\right] \\
& \quad\left\{\begin{array}{l}
e^{-i p_{3}}+e^{i p_{3}} \delta e^{-i \theta} \\
e^{i p_{3}}+e^{-i p_{3}} \delta e^{-i \theta}
\end{array}\right\} A_{n} .
\end{aligned}
$$

With the above solutions, the first-order correction of $\phi$ is

$$
\begin{aligned}
\phi_{1} & =\left[\cos (n \pi y) e^{i k_{n} x}+\left(b_{21} e^{i p_{2} y}+b_{22} e^{-i p_{2} y}\right) e^{i\left(k_{n}+k_{w}\right) x}\right. \\
& \left.+\left(b_{31} e^{i p_{3} y}+b_{32} e^{-i p_{3} y}\right) e^{i\left(k_{n}-k_{w}\right) x}\right] A_{n} .
\end{aligned}
$$

Remember, particle velocity $\widetilde{\vec{v}}=\nabla \Phi$ and sound pressure $\widetilde{p}=$ $-\rho_{0}(\partial \Phi / \partial t)$ and also dependence on time is expressed as $\Phi=$ $\varphi e^{-i \omega t}$ and $u_{x}=-(\partial \varphi / \partial x) e^{-i \omega t} ;$ then

$$
\begin{aligned}
u_{x} & =-\frac{\partial \varphi}{\partial x}=-A_{n}\left[i k_{n} \cos (n \pi y) e^{i k_{n} x}\right. \\
& +i\left(k_{n}+k_{w}\right)\left(b_{21} e^{i p_{2} y}+b_{22} e^{-i p_{2} y}\right) e^{i\left(k_{n}+k_{w}\right) x} \\
& \left.+i\left(k_{n}-k_{w}\right)\left(b_{31} e^{i p_{3} y}+b_{32} e^{-i p_{3} y}\right) e^{i\left(k_{n}-k_{w}\right) x}\right] e^{-i \omega t}, \\
p & =\rho_{0}\left[\frac{\partial \Phi}{\partial t}+\overrightarrow{V_{0}} \cdot \nabla \Phi\right]=\rho_{0}\left[\frac{\partial \varphi e^{-i \omega t}}{\partial t}+\vec{V}_{0}\right. \\
& \left.\cdot \nabla \varphi e^{-i \omega t}\right]=\rho_{0}\left[-i \omega \varphi+\overrightarrow{M_{x}} \cdot \frac{\partial \varphi}{\partial x}\right] e^{-i \omega t} \\
& =-i \omega A_{n}\left[\cos (n \pi y) e^{i k_{n} x}\right. \\
& +\left(b_{21} e^{i p_{2} y}+b_{22} e^{-i p_{2} y}\right) e^{i\left(k_{n}+k_{w}\right) x} \\
& \left.+\left(b_{31} e^{i p_{3} y}+b_{32} e^{-i p_{3} y}\right) e^{i\left(k_{n}-k_{w}\right) x}\right] .
\end{aligned}
$$

Specific acoustic impedance is defined as the ratio of acoustic pressure to the particle velocity; that is, $z=p / u_{x}=r+i x$, where $r$ is specific acoustic resistance, measured in $\mathrm{Pa} \cdot \mathrm{s} / \mathrm{m}$, and $x$ is the specific acoustic reactance, measured in $\mathrm{Pa} \cdot \mathrm{s} / \mathrm{m}$.

2.4. Second-Order Solution. To solve the second-order problem, let us seek a solution for $\phi_{2}$ in the form $\phi_{2}(x, y)=$ $\Psi_{2}(y) e^{i k_{j} x}$, which upon substitution in the system of (12a)(12c) leads to

$$
\begin{aligned}
& \Psi_{2}^{\prime \prime}(y) e^{i k_{n} x}+\left[k_{0}^{2}-k_{n}^{2}\right] \Psi_{2}(y) e^{i k_{n} x}=\left(-k_{1}^{2}-2 k_{0} k_{1}\right. \\
& \left.-2 k_{0} k_{2}\right) A_{n} \cos (n \pi y), \\
& \Psi_{2}^{\prime}(-1)=-\left[\sum_{j=1,3,5, \ldots} \frac{i}{2 j}\right]\left[\left(p_{3}^{2}\right)\left(b_{31} e^{-p_{3}}-b_{32} e^{p_{3}}\right)\right. \\
& \left.-\left(p_{2}^{2}\right)\left(b_{21} e^{-p_{2}}-b_{22} e^{p_{2}}\right)\right]-\left[k_{w} \sum_{j=1,3,5, \ldots} \frac{i}{2 j}\right] \\
& \cdot\left[\left(k_{n}-k_{w}\right)\left(b_{31} e^{-p_{3}}+b_{32} e^{p_{3}}\right)\right. \\
& \left.+\left(k_{n}+k_{w}\right)\left(b_{21} e^{-p_{2}}+b_{22} e^{p_{2}}\right)\right]+\left[\sum_{j=1,3,5, \ldots} \frac{i}{2 j}\right]^{2} \\
& \cdot\left[(n \pi)^{3}(-1)^{n}\right],
\end{aligned}
$$




$$
\begin{aligned}
\Psi_{2}^{\prime}(1)=\left[\delta \sum_{j=1,3,5, \ldots} \frac{i}{2 j}\right] \\
\cdot\left[\left(p_{3}^{2}\right)\left(b_{31} e^{p_{3}}-b_{32} e^{-p_{3}}\right)\left(e^{i \theta}\right)\right. \\
\left.-\left(p_{2}^{2}\right)\left(b_{21} e^{p_{2}}-b_{22} e^{-p_{2}}\right)\left(e^{-i \theta}\right)\right] \\
+\left[\delta k_{w} \sum_{j=1,3,5, \ldots} \frac{i}{2 j}\right] \\
\cdot\left[\left(k_{n}-k_{w}\right)\left(b_{31} e^{p_{3}}+b_{32} e^{-p_{3}}\right)\left(e^{i \theta}\right)\right. \\
\left.+\left(k_{n}+k_{w}\right)\left(b_{21} e^{p_{2}}+b_{22} e^{-p_{2}}\right)\left(e^{-i \theta}\right)\right] \\
+\left[\delta \sum_{j=1,3,5, \ldots} \frac{i}{2 j}\right]^{2}\left[A_{n}(n \pi)^{3}(-1)^{n}\right] .
\end{aligned}
$$

Let us multiply the governing equation (21a) by $\cos (j \pi y)$ and integrate by parts from $y=0$ to $y=1$; then we invoke the boundary conditions (21b) and (21c). This leads to determining the second-order correction of the wavenumber as

$$
k_{2}=\frac{\Psi_{2}^{\prime}(1) \cos (n \pi)-\Psi_{2}^{\prime}(-1)}{2 k_{0}} .
$$

This correction is of significance for estimating the phase speed of the acoustic propagating mode in the undulated pipe, which can be calculated using

$$
c=\frac{\omega}{\operatorname{Re}\left(k_{0}+\varepsilon^{2} k_{2}\right)} .
$$

\section{Discussion and Illustrations}

A solution of the system of (4a)-(4c) in the form of a straightforward first-order asymptotic expansion (without parameter straining) is found to be invalid when two interacting modes whose wavenumbers are $k_{m}$ and $k_{n}$ satisfy the relationship $k_{m} \mp k_{n}=k_{w}$ [1-3]. In the special case of the interaction of two contradirectional acoustic modes, namely, the forwardpropagating mode and its backward-propagating counterpart mode, this condition becomes $2 k_{n}=k_{w}$, which indicates a Bragg resonance. A considerable amount of wave reflection occurs under Bragg resonance as a result of successive interferences with the periodic structures that leads to formation of a stopband and divides the frequency spectrum into patterns of stopbands and passbands. In all calculations in this paper, frequencies located within stopband are surely avoided. In other words, only forward-propagating modes within a passband are focused upon.

In this section, let us consider two acoustic waveguides with rigid walls having square-wave profiles. Both waveguides are assumed to be filled with stationary air, in which the bulk velocity of sound equals $343 \mathrm{~m} / \mathrm{s}$. Both periodic ducts have an average half width $(h)$ of $0.1 \mathrm{~m}$. The ratio between the amplitude of wall corrugation and the average

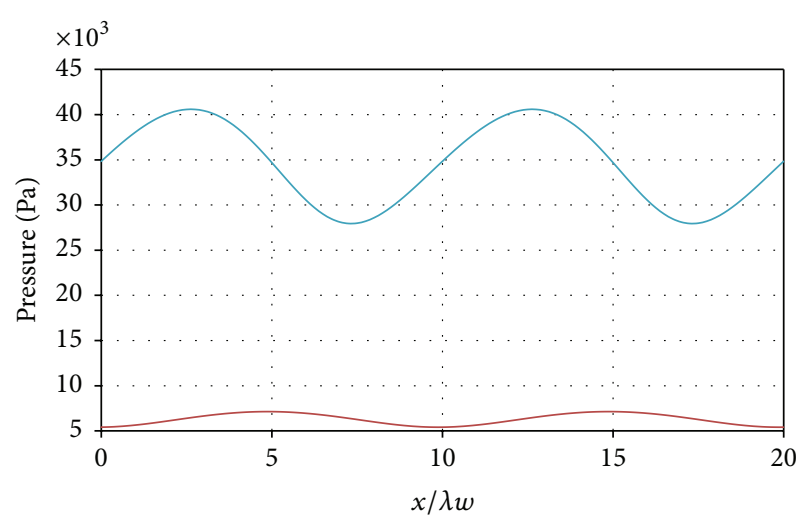

FIGURE 2: Pressure distribution of the fundamental mode at $1 \mathrm{kHz}$ along two acoustic waveguides at $y=0$, one with $k_{w}=1.0$ (blue) and the second with $k_{w}=5.0$ (red), with the parameters $\varepsilon=0.1$, $\theta=90^{\circ}$, and $\alpha=1$.

half width of each duct $(\varepsilon)$ is taken to be 0.1 . The periodic walls of the first and the second ducts have nondimensional wavenumbers $\left(k_{w}\right)$ of 1.0 and 5.0 corresponding to periodic wall wavelengths of $\left(\hat{\lambda}_{w}\right)$ of $0.6283 \mathrm{~m}$ and $0.1257 \mathrm{~m}$, respectively. Each acoustic duct has upper and lower corrugated periodic walls that have a phase angle $(\theta)$ of $90^{\circ}$. Note that the first periodic duct has a low density of wall corrugations while the second has a high density of wall corrugations. For each waveguide, we calculate pressure distribution using (20) and specific acoustic impedance components of resistance and reactance along the duct using the equation $z=p / u_{x}$. Then, we calculate the phase speed of an acoustic signal over a frequency range using (23).

Figure 2 shows the sound pressure distribution along both acoustic waveguides at the waveguide mid-plane $(y=0)$ for the frequency of $1 \mathrm{kHz}$ that is obtained from the equation $\widetilde{p}=-\rho_{0}(\partial \Phi / \partial t)$. It is noted that the acoustic pressure in the first duct is considerably higher than its corresponding sound pressure in the second duct. This can be explained in the light of the fact that the first waveguide represents a "wavy" duct that gives a chance for pressure buildup, while the second waveguide represents a duct with wall "roughness" that marginally blocks the wave leading to low pressure levels. Figures 3(a) and 3(b) show the specific acoustic impedance components (resistance and reactance) along both acoustic waveguides at $1 \mathrm{kHz}$; these are obtained from the equation $z=r+i x$. It is noted that the obtained impedance results go well along the pressure distribution results.

Figure 4 shows the phase speed of an acoustic fundamental mode signal propagating in a duct with walls having high periodic corrugation density (which indicates small wall wavelength and thus exemplifies the case of wall roughness) tends to be higher than the phase speed of acoustic signal traveling in a duct with walls having low periodic corrugation density (which indicates a large wavelength and thus represents the case of weak waviness at the wall).

A further case to consider is a comparison between the acoustic phase speed in a duct having square-wave corrugation and that in a duct having sinusoidal corrugation with 


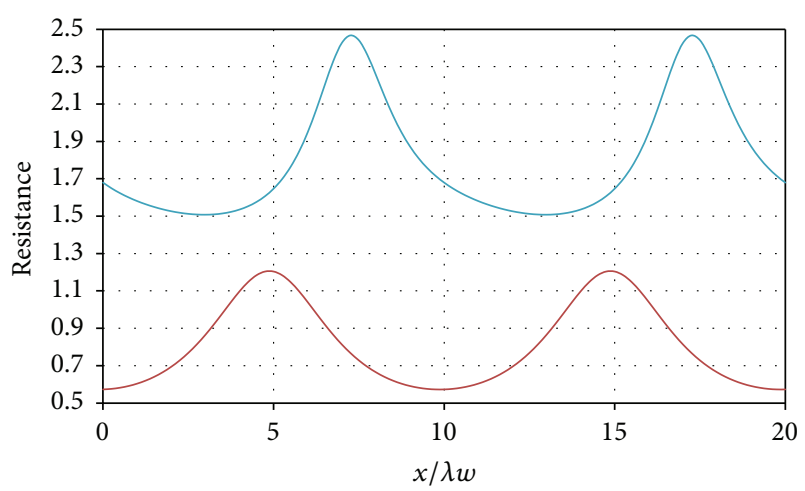

(a)

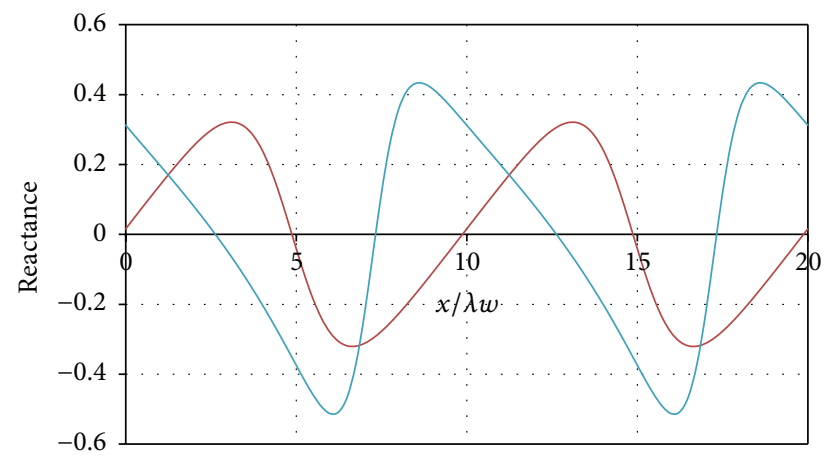

(b)

FIGURE 3: Specific impedance components (resistance and reactance) for the fundamental mode at $1 \mathrm{kHz}$ along two acoustic waveguides, one with $k_{w}=1.0$ (blue) and the second with $k_{w}=5.0$ (red), with the parameters $\varepsilon=0.1, \theta=90^{\circ}$, and $\alpha=1$.

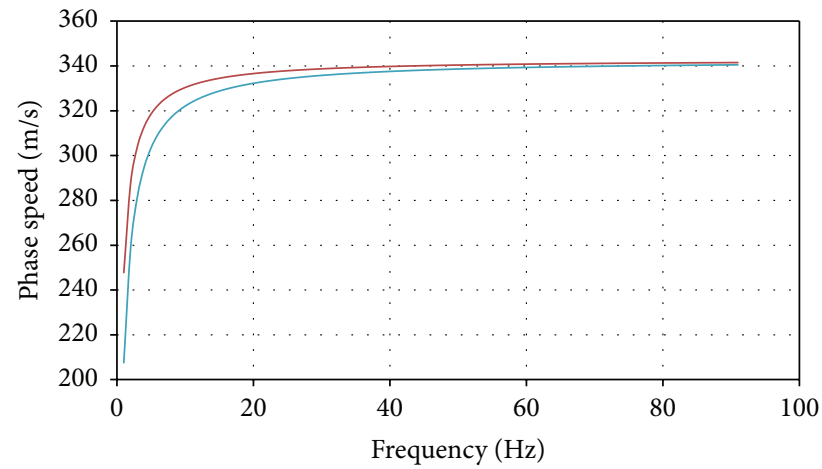

FIGURE 4: Phase speed of the fundamental mode of two acoustic waveguides, one with $k_{w}=1.0$ (blue) and the second with $k_{w}=5.0$ (red), with the parameters $\varepsilon=0.1, \theta=90^{\circ}$, and $\alpha=1$.

the same amplitude of the wall wavelength. Figure 5 shows that the phase speed of the fundamental acoustic propagating mode in the waveguide with square-wave corrugations is slower than that propagating in a waveguide with sinusoidal corrugation. This is an indication that the sharp edges of the square-wave corrugations cause a high level of wave diffraction, which leads to lowering the wave speed.

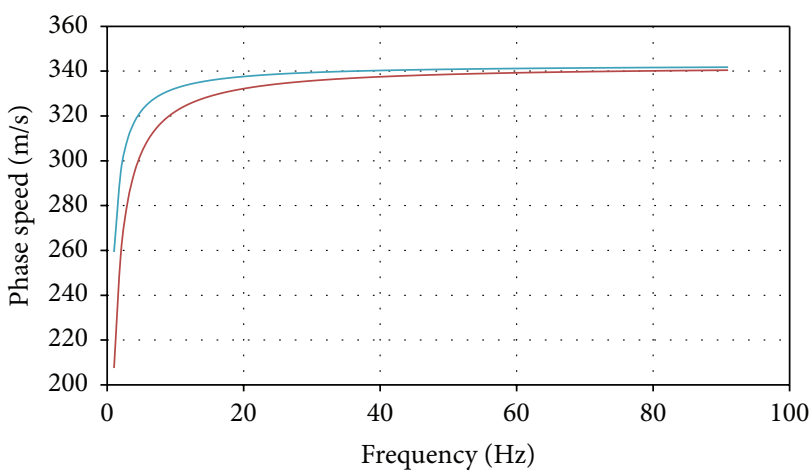

FIGURE 5: Phase speed of the fundamental mode inside two acoustic waveguides, one with sinusoidal wall corrugations having $k_{w}=1.0$ (blue) and the second with square-wave wall corrugations having $k_{w}=1.0$ (red), with the parameters $\varepsilon=0.1, \theta=90^{\circ}$, and $\alpha=1$.

\section{Conclusion}

Acoustic waveguides with hard-wall corrugations having square-wave profile were considered. Since the amplitude of corrugation is small compared to the width of the duct, a small geometric parameter-based perturbation approach was used to describe the acoustic pressure inside the waveguide and to estimate phase speed of acoustic transmitting waves. Acoustic pressure and specific impedance along these waveguides were found to be periodic. The wall corrugation density was found to influence pressure inside periodic waveguides and phase speed of waves transmitting through them. A waveguide with low wall corrugation density is found to have significantly higher pressure values and lower phase speeds than those inside a waveguide with lower wall corrugation density. The phase speeds of waves transmitting in a duct with square-wave wall corrugations were found to be slower than phase speeds of waves transmitting through a duct with sinusoidal wall corrugations.

\section{Conflict of Interests}

The author declares that there is no conflict of interests regarding the publication of this paper.

\section{Acknowledgment}

Research support offered by King Fahd University of Petroleum and Minerals (KFUPM) is acknowledged.

\section{References}

[1] J. C. Samuels, "On propagation of waves in slightly rough ducts," Journal of the Acoustical Society of America, vol. 31, pp. 319-325, 1959.

[2] R. F. Salant, "Wave propagation in periodic structures," Journal of the Acoustical Society of America, vol. 53, pp. 405-507, 1973.

[3] A. H. Nayfeh, "Sound waves in two-dimensional ducts with sinusoidal walls," The Journal of the Acoustical Society of America, vol. 56, no. 3, pp. 768-770, 1974. 
[4] A.-M. Nusayr, "Propagation of waves in rectangular ducts with sinusoidal undulations," Journal of the Acoustical Society of America, vol. 67, no. 5, pp. 1472-1476, 1980.

[5] A. Boström, "Acoustic waves in a cylindrical duct with periodically varying cross section," Wave Motion, vol. 5, no. 1, pp. 59-67, 1983.

[6] O. R. Asfar and A. H. Nayfeh, "The application of the method of multiple scales to wave propagation in periodic structures," SIAM Review, vol. 25, no. 4, pp. 455-480, 1983.

[7] L. Lundqvist and A. Boström, "Acoustic waves in a cylindrical duct with infinite, half-infinite, or finite wall corrugations," Journal of Sound and Vibration, vol. 112, no. 1, pp. 111-124, 1987.

[8] C. E. Bradley, "Time harmonic acoustic Bloch wave propagation in periodic waveguides. Part I. Theory," Journal of the Acoustical Society of America, vol. 96, no. 3, pp. 1844-1853, 1994.

[9] M. A. Hawwa, "Acoustic/elastic stop-band interaction in waveguides involving two periodicities," Journal of the Acoustical Society of America, vol. 102, no. 1, pp. 137-142, 1997.

[10] M. A. Hawwa, "Waveguides with periodic undulations inspired by DSP windowing," Journal of Sound and Vibration, vol. 209, no. 4, pp. 699-706, 1998.

[11] M. A. Hawwa, "Acoustic wave blocking in a duct with a chirped periodic wall," Arabian Journal for Science and Engineering, vol. 29, no. 1, pp. 113-124, 2004.

[12] Z.-Y. Tao, Y.-M. Xiao, and X.-L. Wang, "Non-Bragg resonance of standing acoustic wave in a cylindrical waveguide with sinusoidally perturbed walls," Chinese Physics Letters, vol. 22, no. 2, pp. 394-397, 2005.

[13] C. Potel and M. Bruneau, "Modes coupling due to nonhomogeneously shaped walls in duct acoustics," Journal of Sound and Vibration, vol. 313, no. 3-5, pp. 738-759, 2008.

[14] T. Valier-Brasier, C. Potel, and M. Bruneau, "On the modeling of modes coupling in dissipative fluid-filled waveguide with corrugated surfaces," Journal of Applied Physics, vol. 106, no. 3, Article ID 034913, 2009.

[15] Z. Tao, W. He, Y. Xiao, W. Zhang, and X. Wang, "Sound transmission within the Bragg gap via the high-order modes in a waveguide with periodically corrugated walls," Journal of Applied Physics, vol. 105, no. 12, Article ID 123515, 2009.

[16] S. Del Giudice and G. Bernasconi, "Acoustic response of a sinusoidally perturbed hard-walled duct," Mathematical Problems in Engineering, vol. 2013, Article ID 267291, 6 pages, 2013.

[17] Z.-Y. Tao and Y.-X. Fan, "Resonances of three transverse standing-wave modes in a waveguide with periodic wall undulations," Wave Motion, vol. 51, no. 3, pp. 489-495, 2014.

[18] A. H. Nayfeh, Introduction to perturbation techniques, WileyInterscience, New York, NY, USA, 1981. 


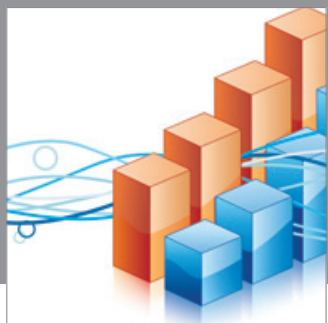

Advances in

Operations Research

mansans

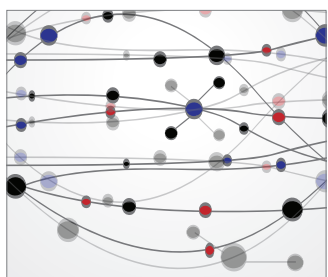

The Scientific World Journal
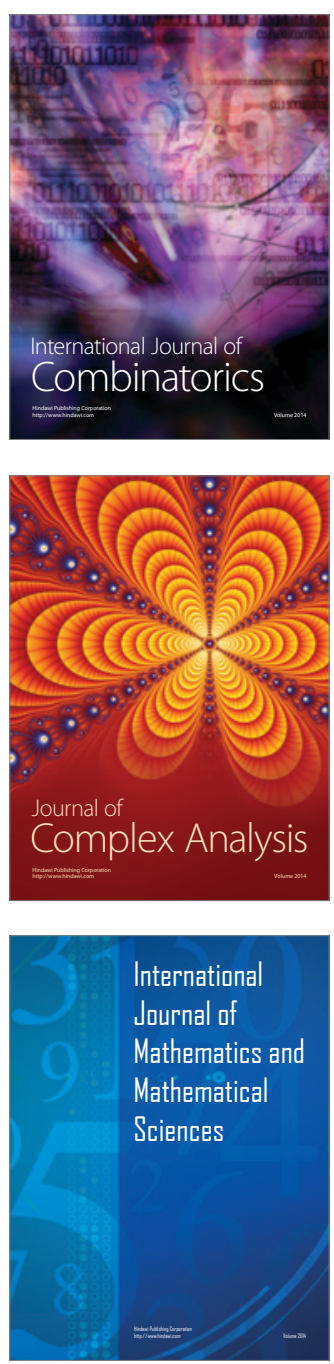
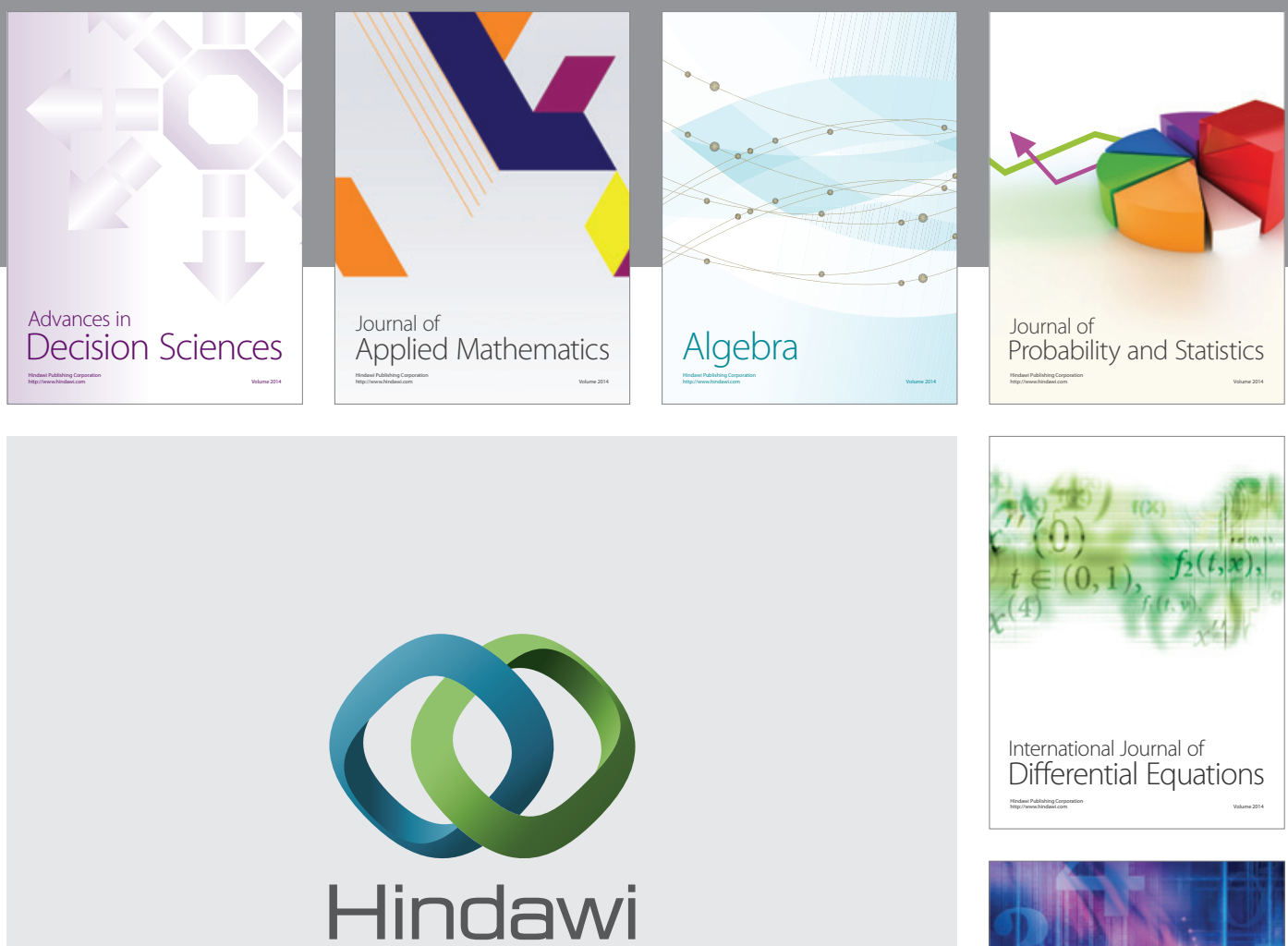

Submit your manuscripts at http://www.hindawi.com
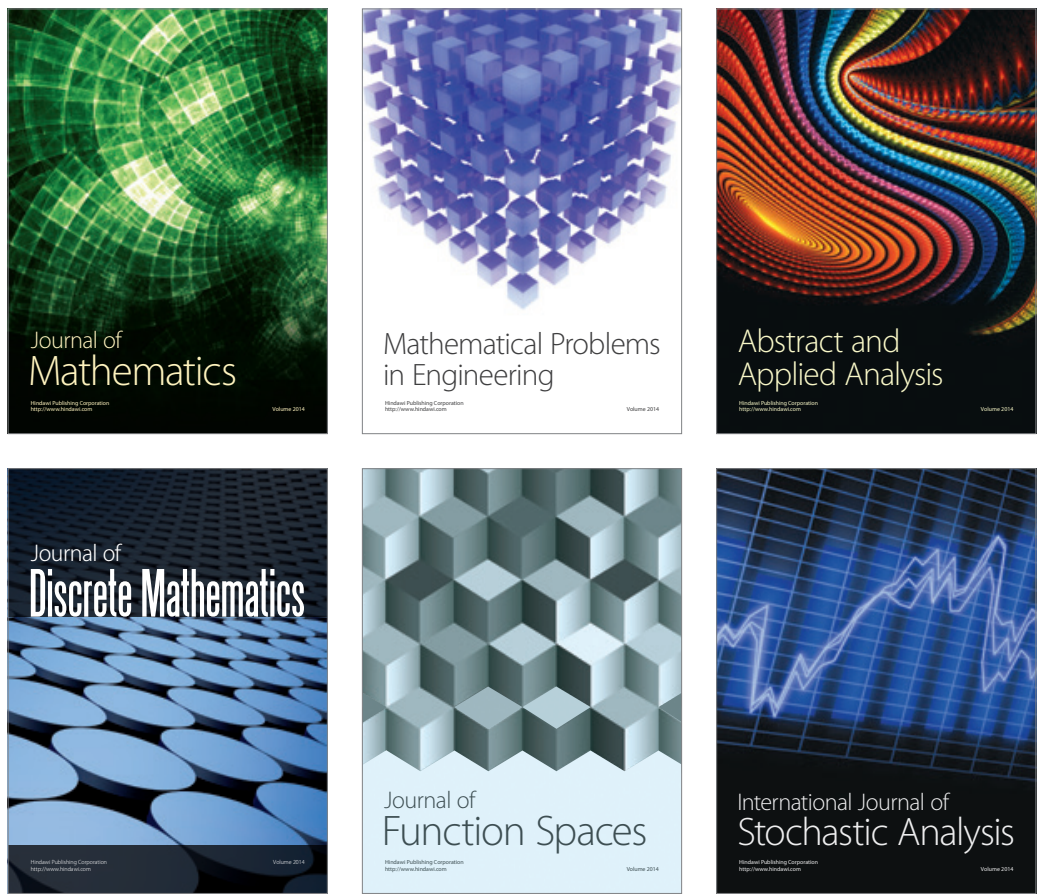

Journal of

Function Spaces

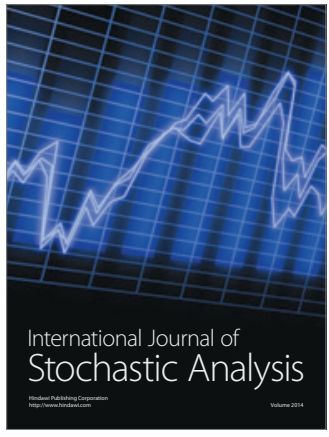

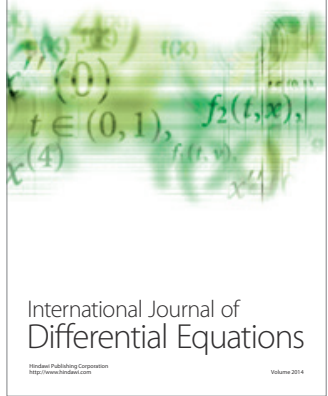
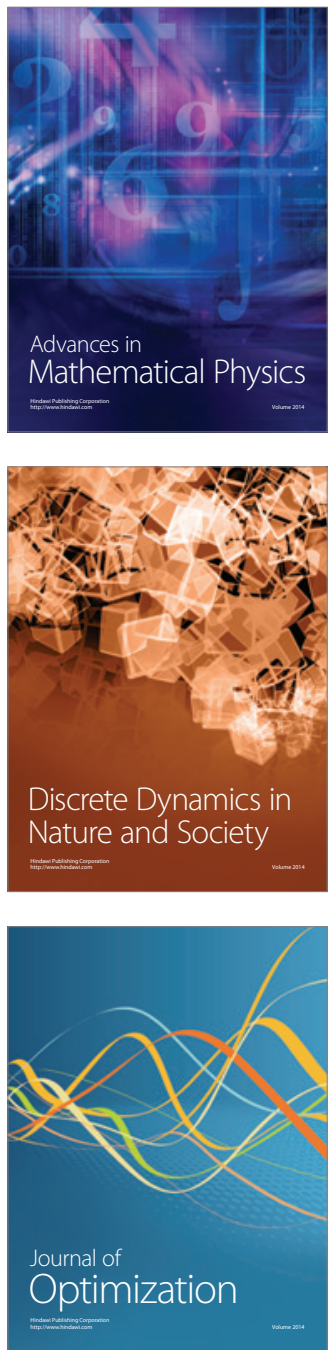